RAL-TR-95-046

September 1995

\title{
Are Diffractive Events at HERA due to a Gluemoron or a Quarkball?
}

\author{
F.E. Close \\ and \\ J.R. Forshaw \\ Rutherford Appleton Laboratory, \\ Chilton, Didcot OX11 0QX, England.
}

\begin{abstract}
We propose a means of distinguishing between gluon- and quark-seeded systems that may in principle be measured in deep inelastic lepton scattering. As a particular application, we discuss those HERA data on deep inelastic ep scattering which contain a large rapidity gap in the final state. It is standard to interpret these events as being due to "pomeron" exchange, although precisely what is meant by this remains unclear. Guided by data, we make some rather general statements about these events and in particular we discuss the potential for discerning whether the exchanged colour singlet system can be interpreted as gluonic.
\end{abstract}


In this letter we propose an experiment that distinguishes between glueballs and conventional quarkbased systems or "quarkballs". The experiment involves deep inelastic lepton scattering and we discuss the application of these ideas to the "pomeron" as revealed in the rapidity gap events which have been observed in electroproduction at HERA [1].

We shall show on rather general grounds that in totally inclusive leptoproduction off a hadron $H$, whose partons carry momentum fractions $\beta$, the $Q^{2}$ dependence of the "momentum" integral,

$$
\frac{d M\left(Q^{2}\right)}{d Q^{2}} \equiv \frac{d}{d Q^{2}} \int_{0}^{1} d \beta F_{2}^{H}\left(\beta, Q^{2}\right)
$$

may be used to distinguish between gluonic or conventional (i.e. quark seeded) hadronic systems. To the extent that the rapidity gap events at HERA enable us to identify a pomeron structure function, i.e. analogous to the $F_{2}^{H}\left(\beta, Q^{2}\right)$ in eq.(1) (and we shall discuss in some detail the feasibility of this) we can apply these ideas to the structure of the pomeron.

The general feature that we shall utilise is that the above integral is related to the fraction of the target's momentum that is carried by electrically charged constituents. The above integral varies with resolution: for a quarkball (such as the proton) the integral in eq.(1) falls gradually to an asymptotic value whereas for a glueball it should rise rather rapidly to its limit [2]. The essential reason, as illustrated in the figure, is that quarks shed momentum by gluon bremsstrahlung whereas gluons feed momentum into $q \bar{q}$, hence in regions of $\beta$ where quarks dominate one will have $\frac{d F_{2}^{H}\left(\beta, Q^{2}\right)}{d Q^{2}}<0$ with increasing $Q^{2}$; by contrast, $\frac{d F_{2}^{H}\left(\beta, Q^{2}\right)}{d Q^{2}}>0$ in regions of gluon dominance. Gluon dominance is anticipated as $\beta \rightarrow 0$ for all systems and hence $\frac{d F_{2}^{H}(\beta \rightarrow 0)}{d Q^{2}}>0$ in general. For a quarkball this is counterbalanced by the behaviour at large $\beta$ where valence quarks dominate: $\frac{d F_{2}^{H}(\beta>0.3)}{d Q^{2}}<0$ such that in the integral $\frac{d M\left(Q^{2}\right)}{d Q^{2}}<0$ overall. For a glueball, however, we would expect that the "valence" gluons cause $\frac{d F_{2}^{H}(\beta>0.3)}{d Q^{2}}>0$ which is quite opposite to that of a quarkball and that a rise should be observed at essentially all values of $\beta$ such that $\frac{d M\left(Q^{2}\right)}{d Q^{2}}>0$.

Data [3, 田 on what has been widely interpreted as the pomeron structure function [5 have been analysed in many models. These models tend to assume either explicitly or implicitly that the pomeron is gluonic and propose tests based on the $\beta$-distributions of its structure function. However, model dependent $\beta$-distributions alone cannot confirm the gluonic hypothesis and our $Q^{2}$ test is a complementary and, we suggest, less model dependent measure.

These general remarks seem not to have been articulated in the literature in this context; we shall now discuss the circumstances under which they follow from the emerging HERA data and then suggest that the data already imply a gluonic pomeron. We first discuss what are the general implications of the rapidity gap events which have been observed in electroproduction at HERA [1] and argue that the data imply that the proton effectively offers up a colour singlet system with which the current interacts. We shall henceforth use "pomeron" as shorthand for "colour singlet that is exchanged" without a priori prejudice as to its internal structure.

On purely kinematical grounds, events in which the incoming proton remains intact and loses only a very small fraction of its initial momentum will be associated with a rapidity gap. The HERA experimentalists are able to measure this (dominant) subset of their rapidity gap events. However, it could in principle be that the proton dissociates into some low mass colour singlet state, rather than remaining intact. These events will 
have essentially the same topology as the ones with a final state proton and good instrumentation in the very forward direction is needed in order to unravel the various components. The HERA experiments are making good progress in this area. However, it should be realised that data published hitherto have been corrected for the background from proton dissociation rather than compiled by direct observation of a final state proton.

Since any exchange of colour between the proton and photon would lead to the production of final state hadrons which would fill in any rapidity gap it follows that the rapidity gap events select those processes in which no net colour is exchanged (between the photon and proton). This is a rather general property, in that nothing is said about the mechanism of the colour neutralisation. For example, the recent work of Buchmüller and Hebecker has the essential dynamics driven by single gluon exchange, the colour neutralisation is supposed to occur sometime after the hard gluon interaction but sometime before the parton hadronisation [6].

There has been much speculation regarding the nature of the dynamics of diffractive DIS. These include the Regge inspired models of refs. [7, 8], the QCD approaches of refs. [9, 10, 11] and the more novel approaches of ref. [6, 12]. Indeed, a substantial fraction of deep inelastic events were predicted to be diffractive (i.e. contain the rapidity gap) using the simple Regge pole ansatz for the pomeron $[7]$.

The cross section,

$$
\frac{d^{4} \sigma\left(x, Q^{2}, x_{P}, t\right)}{d x d Q^{2} d x_{P} d t}=\frac{4 \pi \alpha^{2}}{x Q^{4}}\left(1-y+y^{2} / 2\right) F_{2}^{D}\left(x, Q^{2}, x_{P}, t\right)
$$

defines the (dimensionful) diffractive structure function, $F_{2}^{D}\left(x, Q^{2}, x_{P}, t\right)$, in terms of the Bjorken- $x(x)$, photon virtuality $\left(Q^{2}\right)$, momentum transfer $(t)$ and the variable $x_{P}$, where (ignoring the proton mass)

$$
\begin{aligned}
x & =\frac{Q^{2}}{Q^{2}+W^{2}}, \\
x_{P} & =\frac{Q^{2}+M_{X}^{2}-t}{Q^{2}+W^{2}}, \\
y & =\frac{Q^{2}}{x s} .
\end{aligned}
$$

The $\gamma^{*} p$ and $e p$ invariant masses are $W$ and $\sqrt{ } s$ respectively, and $M_{X}$ is the invariant mass of the (observed) hadronic system associated with the photon dissociation. The variable $x_{P}$ can be interpreted as the fraction of the incident proton momentum which is carried into the interaction.

The experiments do not yet measure $t$, rather they integrate over it and so it is more appropriate to work with the dimensionless function $\tilde{F}_{2}^{D}\left(x, Q^{2}, \tilde{x}_{P}\right)$ defined thus:

$$
\frac{d^{3} \sigma\left(x, Q^{2}, x_{P}\right)}{d x d Q^{2} d \tilde{x}_{P}}=\frac{4 \pi \alpha^{2}}{x Q^{4}}\left(1-y+y^{2} / 2\right) \tilde{F}_{2}^{D}\left(x, Q^{2}, \tilde{x}_{P}\right) .
$$

Note that for $-t \ll Q^{2}+M_{X}^{2}, \tilde{x}_{P} \approx x_{P}$. Since this is usually the case, we will subsequently drop the tilde.

There are two striking empirical properties of the observed $\tilde{F}_{2}^{D}\left(x, Q^{2}, x_{P}\right)$. Firstly, it has only weak $Q^{2}$ dependence and secondly, the $x_{P}$ dependence can be partitioned as follows

$$
\tilde{F}_{2}^{D}\left(x, Q^{2}, x_{P}\right) \approx h\left(\beta, Q^{2}\right) f\left(x_{P}\right)
$$

where

$$
\beta=\frac{x}{x_{P}}
$$


and empirically

$$
f\left(x_{P}\right) \sim x_{P}^{-n}
$$

where $n=1.19 \pm 0.06 \pm 0.07$ (H1) [3] or $n=1.30 \pm 0.08{ }_{-0.14}^{+0.08}$ (ZEUS) 顿. The first error is statistical and the second systematic. It should be appreciated that the errors are large enough to admit an appreciable non-partitioned contribution. The partitioning implies that the production mechanism of the colour singlet is independent of the nature of the deep inelastic probe (it remains to be seen whether the partitioning also applies elsewhere, e.g. in diffractive photoproduction of dijets). To the extent that a fast forward colour singlet proton or excited cluster has been emitted, and at the level of accuracy at which partitioning has been established, we can therefore think of the incoming proton offering up a colour singlet which is then probed by the virtual photon. Thus, the function $h\left(\beta, Q^{2}\right)$ can legitimately be referred to as the structure function of the pomeron [5] ( $\beta$ is then the Bjorken- $x$ defined in the pomeron-photon system) and $f\left(x_{P}\right)$ as the pomeron flux factor. Note that the absolute normalisations of $h\left(\beta, Q^{2}\right)$ and $f\left(x_{P}\right)$ are not well defined, since only their product is measured.

We shall first establish with what restrictions we can impose a parton model interpretation of the structure function, $h\left(\beta, Q^{2}\right)$, i.e.

$$
h\left(\beta, Q^{2}\right)=\sum_{i=1}^{n_{f}} e_{i}^{2} \beta\left[q_{i}\left(\beta, Q^{2}\right)+\bar{q}_{i}\left(\beta, Q^{2}\right)\right] .
$$

and then draw general conclusions about its implications for the microstructure of the colour singlet system.

The weak $Q^{2}$ dependence implies that the diffractive cross section is measuring a leading twist phenomenon and suggests that we can write the structure function $h\left(\beta, Q^{2}\right)$ as a charge weighted sum over parton (quark) densities (i.e. the total cross section is an incoherent sum over individual elastic $\gamma^{*}$-parton cross sections). However, it is important to be clear about what is meant by these parton distribution functions. Universality of the leading-twist parton densities has been proven to all orders in perturbative QCD, (e.g. the quark densities appearing in DIS are the same as those appearing in Drell-Yan) but a key point to appreciate is that both initial and final state interactions generally spoil the property of factorisation of collinear divergences (and hence the concept of a parton density) for any process that is not fully inclusive. Thus factorisation is lost as soon as we look at anything other than the direct products of a hard scattering; in particular the measured proton in diffractive DIS will generally spoil the factorisation properties and hence the notion of pomeron parton densities. However, Nikolaev and Zakharov [10] and Levin and Wüsthoff [1] have demonstrated, to leading $\ln Q^{2}$ accuracy, that it is possible to partition the structure function as in eq.(5) and to write $h\left(\beta, Q^{2}\right)$ in terms of quark probabilities as in eq.(8). Thus, at this level of approximation, we can indeed talk about diffractive parton densities. The possibility or otherwise of consistently implementing next-to-leading $\log Q^{2}$ evolution in diffractive DIS is in our opinion an open question, due to the appearance of factorisation breaking terms.

However, these parton densities will not be universal, since there are interactions which are present in hadronic hard diffraction which do not occur in diffractive DIS. The presence of an extra contribution to hard diffraction in hadron-hadron reactions was recognised by Collins, Frankfurt and Strikman 13 who termed it the "coherent pomeron" contribution (for a more detailed study of the coherent pomeron in hard diffraction see ref.[14]). The presence of the coherent pomeron contribution has been suggested by the UA8 data [15]. Further confirmation of such a contribution (e.g. in diffractive photoproduction of dijets) and the lack of universality 
of diffractive parton densities needs to be established.

To the extent that the structure function $h\left(\beta, Q^{2}\right)$ has been extracted, our test for gluonic or quark-seeded systems may be applied to the pomeron.

A simplistic distinction between these two broad classes of states is that there exists some scale $\tilde{Q}_{0}^{2}$ at which all of the momentum is carried by either quarks or gluons. The concept of constituent parton momentum is not well defined for the pomeron but in general we may define that for a gluonic pomeron the second moment of the parton distributions

$$
M_{S}\left(Q^{2}\right)=\int_{0}^{1} d \beta \beta \sum_{i=1}^{n_{f}}\left(q_{i}\left(\beta, Q^{2}\right)+\bar{q}_{i}\left(\beta, Q^{2}\right)\right),
$$

tends to vanish at some scale $\tilde{Q}_{0}^{2}: M_{S}\left(\tilde{Q}_{0}^{2}\right) \rightarrow 0$. Conversely for an intrinsically pure quark system, the second moment of the gluon density is nugatory (at some scale). With this definition, we can talk about constituent valence partons within the pomeron. It might be that $\tilde{Q}_{0}^{2}$ is small such that we are in a non-perturbative regime, or it might be that $\tilde{Q}_{0}^{2} \gg \Lambda_{Q C D}^{2}$, in which case the pomeron is essentially perturbative: the distinction is not essential for our purposes.

Ideally, we would like to measure the total "momentum" carried by the quarks and anti-quarks as a function of $Q^{2}$, i.e. $M_{S}\left(Q^{2}\right)$. If the pomeron is gluonic, then the total "momentum" carried by the quarks will increase with increasing $Q^{2}$, saturating at asymptotically high $Q^{2}$ to some constant value (whose normalisation depends upon the normalisation of $h\left(\beta, Q^{2}\right)$ ). Fortunately, this property is preserved when we consider $M\left(Q^{2}\right)$, i.e. (see eq.(1))

$$
M\left(Q^{2}\right)=\int_{0}^{1} d \beta h\left(\beta, Q^{2}\right)
$$

The structure function can be written as a linear combination of the singlet and non-singlet structure functions,

$$
M\left(Q^{2}\right)=e_{S}^{2} M_{S}\left(Q^{2}\right)+e_{N S}^{2} M_{N S}\left(Q^{2}\right),
$$

where $M_{N S}\left(Q^{2}\right)$ can be expressed in terms of the non-singlet distribution functions:

$$
M_{N S}^{i j}\left(Q^{2}\right)=\int_{0}^{1} d \beta \beta\left[q_{i}\left(\beta, Q^{2}\right)+\bar{q}_{i}\left(\beta, Q^{2}\right)-q_{j}\left(\beta, Q^{2}\right)-\bar{q}_{j}\left(\beta, Q^{2}\right)\right]
$$

and $e_{j}^{2}$ is the squared charge of the $j$ th quark type. The explicit form for the charge sums and densities depends upon the number of flavours, e.g. for $n_{f}=4$

$$
M\left(Q^{2}\right)=\frac{1}{2}\left(e_{u}^{2}+e_{d}^{2}\right) M_{S}\left(Q^{2}\right)+\frac{1}{2}\left(e_{u}^{2}-e_{d}^{2}\right)\left(M_{N S}^{u d}\left(Q^{2}\right)+M_{N S}^{c s}\left(Q^{2}\right)\right) .
$$

We recall the solutions for the second moments:

$$
\begin{aligned}
M_{N S}\left(Q^{2}\right) & =M_{N S}\left(Q_{0}^{2}\right) X_{N S} \\
M_{S}\left(Q^{2}\right) & =M_{S}\left(Q_{0}^{2}\right) X_{S}+\frac{3 n_{f}}{16+3 n_{f}}\left(1-X_{S}\right) \mathcal{N}
\end{aligned}
$$

where

$$
\begin{aligned}
X_{N S} & =\exp \left(\frac{32}{3\left(33-2 n_{f}\right)} \ln \frac{\alpha_{s}\left(Q^{2}\right)}{\alpha_{s}\left(Q_{0}^{2}\right)}\right) \\
X_{S} & =\exp \left(\frac{2\left(16+3 n_{f}\right)}{3\left(33-2 n_{f}\right)} \ln \frac{\alpha_{s}\left(Q^{2}\right)}{\alpha_{s}\left(Q_{0}^{2}\right)}\right)
\end{aligned}
$$


and

$$
M_{S}\left(Q^{2}\right)+M_{N S}\left(Q^{2}\right)=\mathcal{N}
$$

We treat $\mathcal{N}$ as an arbitrary factor, which fixes the normalisation of $h\left(\beta, Q^{2}\right)$. Thus,

$$
\begin{aligned}
\frac{d M\left(Q^{2}\right)}{d \ln Q^{2}} & =\frac{X_{S}}{\ln Q^{2} / \Lambda^{2}} \frac{2\left(16+3 n_{f}\right)}{3\left(33-2 n_{f}\right)}\left(\frac{3 \mathcal{N} n_{f}}{16+3 n_{f}}-e_{S}^{2} M_{S}\left(Q_{0}^{2}\right)\right) \\
& -\frac{X_{N S}}{\ln Q^{2} / \Lambda^{2}} \frac{32}{3\left(33-2 n_{f}\right)} e_{N S}^{2} M_{N S}\left(Q_{0}^{2}\right) .
\end{aligned}
$$

We can now show that if

$$
\frac{d M\left(Q^{2}\right)}{d \ln Q^{2}}>0
$$

then the pomeron is gluonic. The truth of the above inequality implies that

$$
M_{S}\left(Q_{0}^{2}\right)<\frac{3 \mathcal{N} n_{f}}{16+3 n_{f}} \frac{1}{e_{S}^{2}}-\frac{16}{16+3 n_{f}} \frac{X_{N S}}{X_{S}} \frac{e_{N S}^{2}}{e_{S}^{2}} M_{N S}\left(Q_{0}^{2}\right)
$$

Since $X_{N S}\left(Q_{1}^{2}\right) / X_{S}\left(Q_{1}^{2}\right)<X_{N S}\left(Q_{2}^{2}\right) / X_{S}\left(Q_{2}^{2}\right)$ for $Q_{1}^{2}<Q_{2}^{2}$ it therefore follows that if eq.(17) holds true at some value of $Q^{2}$ it must also hold at all lower values of $Q^{2}$. Hence there exists some scale, $\tilde{Q}_{0}^{2}$, such that $M\left(\tilde{Q}_{0}^{2}\right) \rightarrow 0$ and so the system is gluonic.

Since the inequality (eq.(17)) completely defines the gluonic system, it follows that a quark-seeded state satisfies the opposite inequality, i.e.

$$
\frac{d M\left(Q^{2}\right)}{d \ln Q^{2}}<0
$$

Note that our definition of quark-/gluon-seeded only requires a measurement at some perturbative scale, $Q^{2} \gg$ $\Lambda_{Q C D}^{2}$. It is not expected that the detailed and essentially unknown non-perturbative physics will spoil our conclusions.

We have implicitly assumed that the pomeron does not have a pointlike, direct coupling to individual quarks, e.g. like the photon. Any such coupling would automatically lead to a contribution which satisfies eq.(17) and hence could fake a gluonic pomeron. Note that a large pointlike coupling of the pomeron can arise if it is assumed that the pomeron interacts essentially as a single gluon, e.g. at some scale the pomeron has a gluon density which is a delta function at $x_{P}$ whilst the quark density is zero. Such a scenario is the extreme case of a super-hard gluonic pomeron and as such will satisfy eq.(17). This is the basic idea of the model of ref.[6]. Any other (i.e. anomalous) pointlike coupling would be of remarkable interest in itself but remains at the level of speculation [12]. Note that the model of Donnachie-Landshoff does allow the pomeron to have a non-pointlike, direct coupling to quarks, i.e. coupling to off-shell quarks is suppressed, by a form factor, at a rate which is characteristic of the pomeron radius. Such a coupling kills off the leading logarithmic contribution and does not, at leading $\log Q^{2}$, affect the $Q^{2}$ evolution.

We recall that, to the extent that colour singlet exchange drives the diffractive events, one may anticipate photoproduction of glueball type hadrons in the central region. In $p p \rightarrow p_{f}(X) p_{s}$ (where $p_{f, s}$ denote forward and spectator protons) the centrally produced $X$ is hypothesised to be glueball rich if it is produced by pairs of colour singlet gluonic pomerons, or "gluemorons" [16]. Similar phenomena may be expected in the diffractive events at HERA if these are due to gluemorons. Such isolated central production is less clear in models of the Buchmuller type [6] and the implications of this as a possible discriminator merit further investigation. 
In the case of quark hadrons, such as the proton, the sign of $\frac{d M\left(Q^{2}\right)}{d \ln Q^{2}}$ tends to be hidden in the errors due to the counterbalancing rise at small $x$ and fall at large $x$ where the valence quarks dominate (fig.1(a)). A similar situation holds for the pion and we note that it will be an interesting test of our general procedure to isolate the pion cloud in $\gamma^{*} p \rightarrow n+X$. For a gluonic system one may therefore anticipate a sharper signal since the valence gluons will drive a rise in $h\left(\beta, Q^{2}\right)$ across the entire range of $\beta$ and hence $\frac{d M\left(Q^{2}\right)}{d \ln Q^{2}}>0$ (fig.1(b)). The diffractive events at HERA seem already to manifest this phenomenon (i.e. a rise across the entire $\beta$-range) [3, 4. Although the error bars are as yet too large to establish that the pomeron structure function is exhibiting a rise with increasing $Q^{2}$ regardless of $\beta$, the data are certainly not falling with $Q^{2}$ in the "valence" region, $\beta \sim 0.65$. This already contrasts with nucleon structure functions where the valence quarks are shedding momentum even for $x$ (" $\beta$ ") as small as 0.3 . Thus barring some radically new behaviour at large $\beta$ (which would be at least as interesting as all that has gone before) the result $\frac{d M\left(Q^{2}\right)}{d \ln Q^{2}}>0$ seems possibly to be established qualitatively already. As such this could well be the first evidence for the presence of colour singlet gluonic systems.

\section{References}

[1] M.Derrick et al (ZEUS Collaboration), Phys.Lett. B315 (1993) 481;

T.Ahmed et al (H1 Collaboration), Nucl.Phys. B429 (1994) 477.

[2] F.E.Close, R.G.Roberts and G.G.Ross, Phys.Lett. B183 (1987) 101.

[3] T.Ahmed et al (H1 Collaboration), Phys.Lett. B348 (1995) 681.

[4] M.Derrick et al (ZEUS Collaboration), DESY 95-093 (Zeit.Phys. to appear).

[5] G.Ingelman and P.Schlein, Phys.Lett. B152 (1985) 256;

[6] W.Buchmüller, Phys.Lett. B353 (1995) 335;

W.Buchmüller and A.Hebecker, Phys.Lett. B355 (1995) 573.

[7] A.Donnachie and P.V.Landshoff, Phys.Lett. B191 (1987) 309; Nucl.Phys. B303 (1988) 634.

[8] A.Capella et al, Phys.Lett. B343 (1995) 403; LPTHE Orsay 95-33;

T.Gehrmann and W.J.Stirling, DTP/95/26-REV;

K.Golec-Biernat and J.Kwieciński, Phys.Lett. B353 (1995) 329;

R.Fiore, L.L.Jenkovszky and F.Paccanoni, ITP-94-44-E, HEP-PH-9505284;

K.Goulianos, RU 95/E-26 (1995), HEP-PH-9505310.

[9] J.Bartels and G.Ingelman, Phys.Lett. B235 (1990) 175;

M.G.Ryskin, Sov.J.Nucl.Phys. 52 (1990) 529;

J.Bartels and M.Wüsthoff, Zeit.Phys. C66 (1995) 157.

[10] N.N.Nikolaev and B.G.Zakharov, Zeit.Phys. C53 (1992) 331; C64 (1994) 631. 
[11] E.Levin and M.Wüsthoff, Phys.Rev. D50 (1994) 4306.

[12] B.A.Kniehl, H.-G.Kohrs and G.Kramer, Z.Phys. C65 (1995) 657.

[13] J.C.Collins, L.Frankfurt and M.Strikman, Phys.Lett. B307 (1993) 161.

[14] A.Berera and D.E.Soper, Phys.Rev. D50 (1994) 4328.

[15] A.Brandt et al (UA8 Collaboration), Phys.Lett. B297 (1992) 417.

[16] S.Abatzis et al, Phys Lett. B324 (1994) 509;

D.Alde et al, Phys Lett. B201 (1988) 160;

C.Amsler and F.E.Close, Phys.Lett. B353 (1995) 385. 


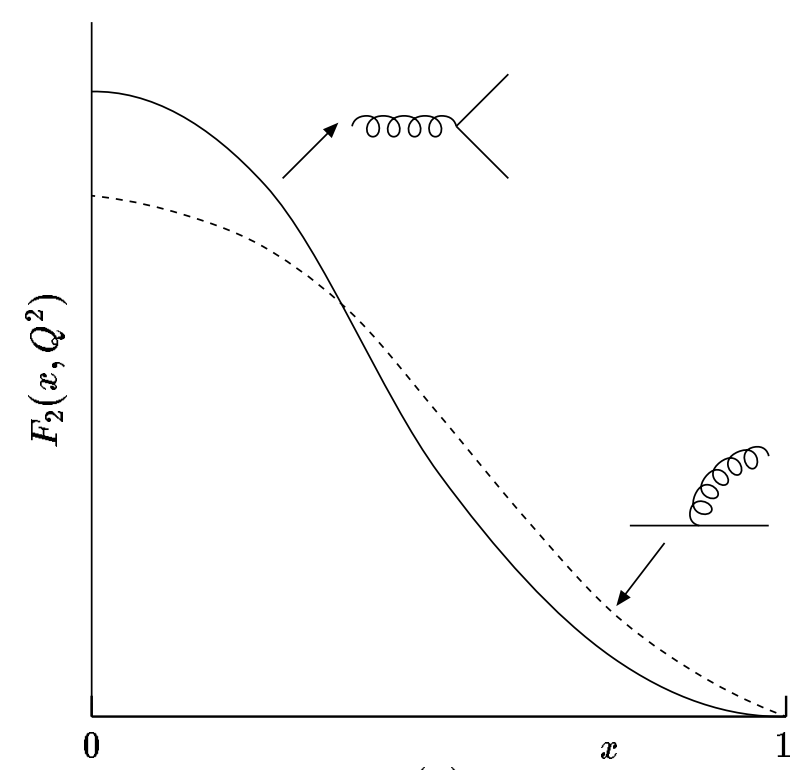

(a)

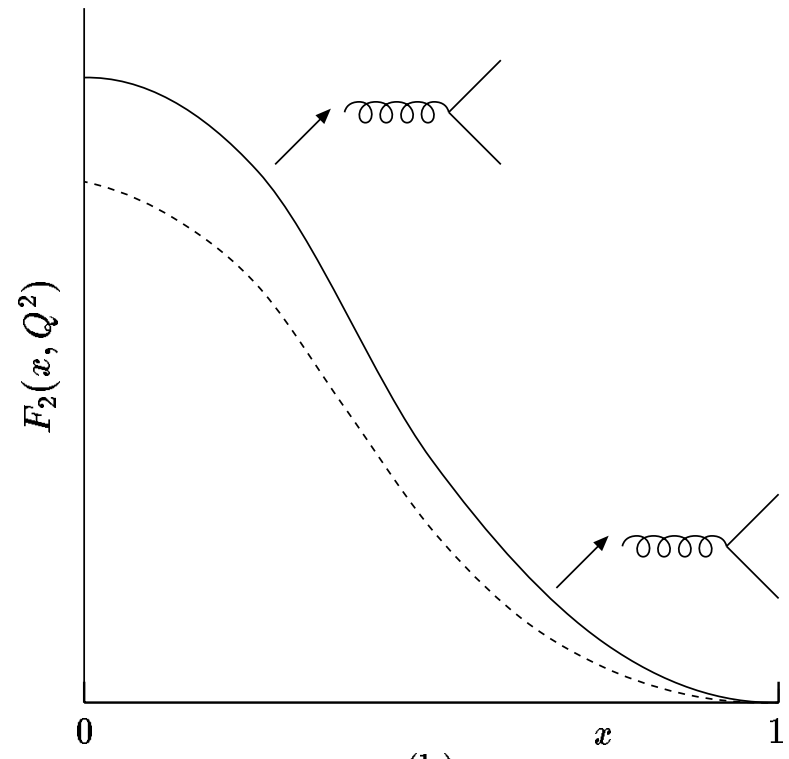

(b)

Figure 1: Schematic 'Quarkball' and 'Glueball' evolution of $F_{2}\left(x, Q^{2}\right)$. Increasing $Q^{2}$, the quarkball (a) rises at small $x$ and falls at large $x$ (due to the radiation shown in the cartoons). The glueball (b) rises across the whole $x$ range. 